\title{
Proceedings of the 2010 Symposium on Hangover and Other Residual Alcohol Effects: Predictors and Consequences
}

\author{
Jonathan Howland ${ }^{*}, 1$, Damaris J. Rohsenow ${ }^{2}$, John E. McGeary ${ }^{2}$, Chris Streeter $^{3}$ and \\ Joris C. Verster ${ }^{4}$ \\ ${ }^{I}$ Department of Community Health Sciences and Department of Emergency Medicine, Boston University, Boston, MA \\ 02118 , USA \\ ${ }^{2}$ Providence Veterans Affairs Medical Center and Center for Alcohol and Addiction Studies at Brown University, \\ Providence, RI 02912, USA \\ ${ }^{3}$ Department of Psychiatry, Boston University, Boston, MA 021184, USA \\ ${ }^{4}$ Utrecht Institute for Pharmaceutical Sciences, Division of Pharmacology, Utrecht University, Utrecht, The Netherlands
}

Keywords: Alcohol, hangover, sensitivity, genetics, brain activity.

\section{INTRODUCTION}

For the second time in 2010, members of the Alcohol Hangover Research Group and others gathered for a symposium on findings from recent studies of alcohol hangover. The first meeting, organized by Joris Verster, Ph.D, and Richard Stephens, Ph.D., was held in June 2010 at the $33^{\text {rd }}$ annual scientific meeting of the Research Society on Alcoholism.

The importance of hangover research lies in its effects on student and worker performance and the implications of hangover-related impairment for safety and worker productivity. Few previous hangover studies used rigorous laboratory methods that controlled with matched placebo, ensured that next-day performance was measured when BAC was at or near zero, and used validated hangover measures. Recently, consensus was reached on methodological issues of alcohol hangover research [1].

A series of hangover studies were conducted over the last decade by the Boston University/Brown University research group. The symposium presentations had a set of common methodologies, including alcohol administration to 0.11 to $0.12 \mathrm{~g} \%$ breath alcohol level (BrAC), matched placebo on a different night (order counterbalanced), ensuring 8 hours with lights out in sleeping quarters, ensuring zero breath alcohol the next morning before testing, and using a hangover measure with only validated items. All participants had no past history of alcohol abuse or dependence and were heavy episodic drinkers.

\section{Hangover Sensitivity in College Does Not Predict} Transition to post-College Drinking

Given that $25-30 \%$ of heavy drinkers do not experience hangover, and given that a minority of heavy drinkers

*Address correspondence to this author at the Department of Community Health Sciences, Boston University School of Public Health, 801 Massachusetts Avenue, Crosstown Center $-3^{\text {rd }}$ Floor, Boston, MA 02118, USA; Tel: 617638 5158; Fax: 617638 4483; E-mail: jhowl@bu.edu continue heavy drinking into the post-college years, this preliminary study investigated the degree to which hangover sensitivity predicted transition to post-college drinking. The study followed 134 undergraduates who had participated in a study of the residual effects of intoxication on next-day performance and were re-contacted 1-5 years postgraduation. In the original study (Time 1), information was collected on participants' drinking practices, alcohol was administered to $0.12 \mathrm{~g} \% \mathrm{BrAC}$ on average, and intensity of hangover was assessed the next morning. Participants were re-contacted $1-4$ years later $($ mean $=2.3$ years, Time 2$)$ to complete a web-based survey of post-college drinking and drinking-related problems. Regression analyses controlled for age, gender, and employment at the later time and Time 1 drinking practices in predicting alcohol quantity/frequency and problems at Time 2. Results indicated that university drinking practices, not hangover incidence or severity, were a strong predictor of transitional drinking $(\mathrm{p}<.0001)$ but there was a trend for hangover to predict fewer alcohol problems at Time $2(\mathrm{p}<.09)$. College drinking rather than hangover is thus the stronger predictor of continued heavy drinking and risk of problems after university.

\section{Hangover Sensitivity Predicts Residual Alcohol Effects on Attention Reaction Time}

The next study examined the relationship of hangover to deficits in a measure of sustained attention combined with reaction time the morning after alcohol intoxication. Participants randomly received bourbon or vodka to a mean peak $\mathrm{BrAC}$ of $0.11 \mathrm{~g} \%$ and, after an 8 -hour period for sleep, completed a validated hangover scale and an attention/ reaction time test (Psychomotor Vigilance Task [PVT] median reaction time) when BrAC was at or near zero. Two regression analyses were used to predict reaction time scores using hangover severity (total score of the Acute Hangover Scale) in one model and hangover incidence (hangover yes/no) in another model. Both models controlled for sex, alcoholic beverage type, average drinking volume, and PVT score under placebo condition. Hangover incidence showed a 
trend towards significance $(\mathrm{p}=.057)$ and hangover severity was a significant $(\mathrm{p}<.05)$ predictor of PVT scores. These findings suggest that hangover is either a mediator of residual alcohol effects or that some biological effect of heavy drinking produces both hangover and impairment. A number of possible factors were reviewed. It was suggested that future studies of residual alcohol effects account for the relationship of hangover to nextday impairment.

\section{Residual Effects of Intoxication on Brain Activity During Psychomotor Vigilance Task Performance: fMRI Results}

This study compared prefrontal, temporal and parahippocampal activation during a measure of sustained attention combined with reaction time, the Continuous Performance Task (CPT) to assess whether brain activation differed in the hangover condition compared to the placebo condition. Participants were eight college students or recent graduates (age range: 21-30) who had reported hangover in a previous laboratory induction and who returned for this additional study. Participants randomly received an alcoholic or placebo beverage one night and the other beverage the other night, with $M=0.12 \mathrm{~g} \% \mathrm{BrAC}$ after alcohol, and an 8-hour sleep opportunity. CPT was chosen due to previous hangover effects and known areas of activation. The few errors were removed prior to performance analysis. Blood oxygen level dependent (BOLD) images were acquired on a Philips Intera 3T scanner and processed in Brain Voyager QX Version 2.1 and mapped into Talairach space for group analysis. Each participant's data was analyzed using a fixed-effect General Linear Model (GLM). Differences were calculated in the activation for correct responses for the alcohol minus the placebo condition. A significant increase in activation was seen during the hangover condition in the following regions: orbital frontal $(\mathrm{p}<0.001)$, dorsolateral $(\mathrm{p}<0.001)$, temporal $(\mathrm{p}<$ $0.001)$ and hippocampus $(p<0.001)$. In the hangover condition, participants were able to perform the task correctly the majority of the time, but the maintenance of a correct performance was accomplished during compensatory recruitment of prefrontal and temporal structures. This study suggests that performing correctly requires more mental effort when hungover.

\section{Genetic Variation Associates with Hangover the Morning After Drinking to Intoxication: Analyses of Alcohol Dehydrogenase Polymorphisms}

The next study focused on genetic predictors of hangover. Variation in the genes for alcohol dehydrogenase (ADH) enzymes may relate to hangover as the gene products metabolize both ethanol itself and the congener methanol. Similarly, variation in genes for aldehyde dehydrogenase (ALDH) may relate to hangover by increasing buildup of acetaldehyde. The only previous study of the role of ADH genes used only a retrospective survey of hangover. The purpose of this study was to investigate this question after inducing hangover and using the Acute Hangover Scale. Participants engaged in several larger laboratory studies of hangover were contacted to contribute buccal cells for DNA analysis. Participants performed attention/reaction time testing and completed the Acute Hangover Scale after an evening of drinking to $0.12 \mathrm{~g} \%$ $\mathrm{BrAC}$ and again after drinking placebo. Five single nucleotide polymorphisms (SNPs) in the ADH cluster on chromosome 4 were tested for association with scores on the hangover scale and neurocognitive performance on the morning after alcohol consumption. Variation in the ADH1C gene was associated with the subjective report of hangover and the sum symptoms score on the hangover scale but not with neurocognitive performance. Haplotype analyses showed the clearest picture of results. These findings suggest that variation in the alcohol metabolizing genes may be associated with a greater sensitivity to the residual effects of intoxication on subjective report of hangover. Variants of ADH1C that are associated with risk for alcohol dependence are more frequent in those who do not report hangover after drinking to intoxication, suggesting a protective effect on hangovers and possibly a protective effect of hangovers on development of alcohol dependence.

\section{Concluding Remarks}

The findings presented at the ISBRA symposium underscore the scope of hangover as a public health issue. From different angles, it was made clear that alcohol hangover has serious socioeconomic and health consequences. Future research is therefore essential. This research should further focus on examining the pathology of alcohol hangover and explore the determinants of risk. This knowledge may enable development of effective prevention strategies and treatment for the alcohol hangover.

\section{ACKNOWLEDGMENTS}

This symposium and the work presented were supported by: a Senior Research Career Scientist Award from the Department of Veterans Affairs, an award from National Institute on Alcohol Abuse and Alcoholism \# P60 AA013759 (Youth Alcohol Prevention Center, Boston University School of Public Health), and by the National Center for Research Resources (NCRR), a component of the National Institutes of Health, through an award \# M01 RR00533. We invite interested parties to join the Alcohol Hangover Research Group (www.alcoholhangover.com).

\section{REFERENCES}

[1] Verster JC, Stephens R, Penning R, et al. The Alcohol hangover research group consensus statement on best practice in alcohol hangover research. Curr Drug Abuse Rev 2010; 3: 116-27. 